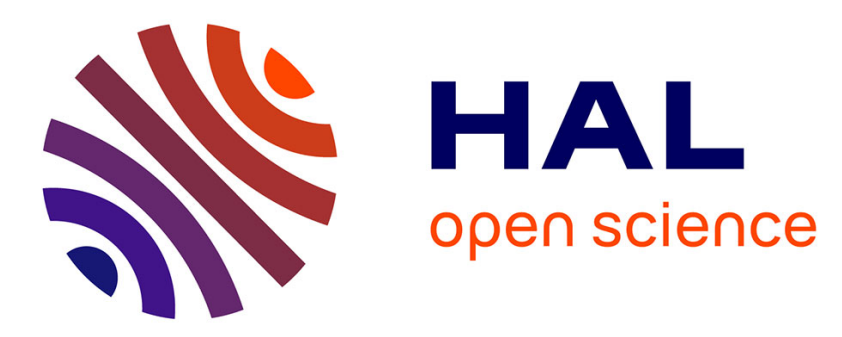

\title{
Delay-Scheduled State-Feedback Design for Time-Delay Systems with Time-Varying delays
}

Corentin Briat, Olivier Sename, Jean-François Lafay

\section{To cite this version:}

Corentin Briat, Olivier Sename, Jean-François Lafay. Delay-Scheduled State-Feedback Design for Time-Delay Systems with Time-Varying delays. IFAC WC 2008 - 17th IFAC World Congress, Jul 2008, Séoul, South Korea. hal-00336982

\section{HAL Id: hal-00336982 https://hal.science/hal-00336982}

Submitted on 17 Jun 2010

HAL is a multi-disciplinary open access archive for the deposit and dissemination of scientific research documents, whether they are published or not. The documents may come from teaching and research institutions in France or abroad, or from public or private research centers.
L'archive ouverte pluridisciplinaire HAL, est destinée au dépôt et à la diffusion de documents scientifiques de niveau recherche, publiés ou non, émanant des établissements d'enseignement et de recherche français ou étrangers, des laboratoires publics ou privés. 


\title{
Delay-Scheduled State-Feedback Design for Time-Delay Systems with Time-Varying Delays
}

\author{
C. Briat* O. Sename* J.F. Lafay** \\ * GIPSA-Lab, Departement of Control Systems (former LAG), \\ Grenoble Universités, ENSIEG - BP46, 38402 Saint Martin d'Hres - \\ Cedex FRANCE, \{ Corentin.Briat, Olivier.Sename\}@gipsa-lap.inpg.fr \\ ** IRCCyN - Centrale de Nantes, 1 rue de la Nö̈ - BP 92101, 44321 \\ Nantes Cedex 3 - FRANCE, Jean-Francois.Lafay@irccyn.ec-nantes.fr
}

\begin{abstract}
This paper is concerned in the synthesis of delay-scheduled state-feedback stabilizing linear systems with time-varying delay when the delay can be approximatively known in realtime. First we introduce a new model transformation turning the time-delay system into an uncertain LPV system. Using this reformulation we elaborate delay-dependent stability test based on the so-called full block $\mathcal{S}$-procedure and derive from it a delay-dependent stabilization lemma. Our results are then relaxed using a new relaxation lemma which is shown to have good properties and provide then LMI based theorems, well-known for their tractability. Our results tackle error measurement on the delay. We show the efficiency of the method through an example.
\end{abstract}

Keywords:

Systems with time-delays; Linear parameter-varying systems; Robust linear matrix inequalities

\section{INTRODUCTION}

Since several years, time-delay systems (TDS) have been intensively studied [Niculescu, 2001, Gu et al., 2003, Fridman, 2006, Gouaisbaut and Peaucelle, 2006b, Suplin et al., 2006, Kao and Rantzer, 2007]. Indeed, delays are often responsible of instability and poor performances and their effect must be considered to analyze stability and synthesize control laws. Since the advent of electrical communication networks and embedded electronics, system with time-varying delays have gained more and more interests since the control input and the measured output of a system controlled through network are affected by a timevarying delay physically corresponding to the information propagation. There also exist many engineering problems where time-varying delays are involved such as milling process...

In some applications, it may be possible to measure or compute the delay from a mathematical model and in this case, it is interesting to use it in the controller. In [Witrant et al., 2005], a predictive approach to control NCS is given but a network model is necessary to compute the prediction horizon. In [Sename et al., 1995] a state feedback with internal delay is designed but the robustness issue w.r.t delay measurement uncertainties is not considered. The authors have proposed in [Briat et al., 2007] some preliminary results on control design for LPV TDS. Using some new model transformation, the time-delay system is transformed into an uncertain Linear Parameter Varying (LPV) system where the delay acts now as a parameter and not anymore as an operator. However the results did only stand for interval delay (with non zero delay). Some interesting results on similar Linear Fractional Transformation (LFT) transformations may also be found in [Zhang et al., 2001, Roozbehani and Knospe, 2005, Gouaisbaut and Peaucelle, 2006a, Kao and Rantzer, 2007].

This paper follows [Briat et al., 2007], the LPV/uncertain system stability analysis and control synthesis tools are used to prove stability and stabilize time-delay systems (See [Apkarian and Adams, 1998, Scherer, 1999]). The main contributions of the paper are:

- Following the path of [Briat et al., 2007] we propose here a new model transformation correcting the weaknesses of the previous one.

- We propose then a method to approximate LMI with polynomial dependence onto parameters into LMI with a simple linear parameter dependence. This result is based on spectral factorization and wellknown lemmas used in matrix algebra. This results is closely related to SOS representation and relaxation (see [Scherer and Hol, 2006, Dietz et al., 2006]).

- Using these results we provide a delay-dependent/ratedependent stability $/ \mathcal{H}_{\infty}$-performances test using the so-called full-block $\mathcal{S}$-procedure and preliminary results of the paper. Computational approximations are proposed and fully explained.

- From this stability test, we derive a stabilization lemma expressed independently of the controller. The obtained controlled is smoothly scheduled by the delay value and take into account a priori bounds on error measurements. It may be computed either using an explicit formulation or via SDP with some addi- 
tional constraints. The originality of our approach allows to consider several costs with different trade-offs depending explicitly of the delay and its derivative.

- We show the validity through several examples.

We consider in this paper system of the form

$$
\begin{aligned}
& \dot{x}(t)=A x(t)+A_{h} x(t-h(t))+B_{u} u(t)+E w(t) \\
& z(t)=C x(t)+C_{h} x(t-h(t))+D_{u} u(t)+F w(t)
\end{aligned}
$$

where $x \in \mathbb{R}^{n}, u \in \mathbb{R}^{m}, w \in \mathbb{R}^{p}$ and $z \in \mathbb{R}^{q}$ are respectively the system state, the control input, the exogenous input and the controlled output.

The time-varying delay $h(t)$ is assumed to belong to the set

$$
\mathscr{H}:=\left\{h \in \mathcal{C}^{1}\left(\mathbb{R}_{+}, H\right), h^{\prime}: \mathbb{R}_{+} \rightarrow U\right\}
$$

where $\mathcal{C}^{1}(I, J)$ denotes the set of continuous functions with continuous derivative mapping $I$ to $J, H:=\left[h_{\min }, h_{\max }\right]$ and $U:=\left[\mu_{\min }, \mu_{\max }\right]$.

We define the vertices set as

$$
\begin{aligned}
& \mathcal{V}_{h}:=\left\{h_{\text {min }}, h_{\text {max }}\right\} \\
& \mathcal{V}_{\mu}:=\left\{\mu_{\text {min }}, \mu_{\text {max }}\right\}
\end{aligned}
$$

The aim of the paper is to find delay-scheduled statefeedback controllers of the form

$$
u(t)=K(\hat{h}(t)) x(t)
$$

where $\hat{h}(t)=h(t)+\delta_{h}(t)$ is the known value of the delay while $\delta_{h}(t)$ is the knowledge error at time $t$ belonging to

$$
\Delta:=\left\{\delta_{h}: \mathbb{R}_{+} \rightarrow \Delta, \delta_{h}^{\prime}: \mathbb{R}_{+} \rightarrow \Delta_{\nu}\right\}
$$

where $\Delta:=[-\delta, \delta]$ and $\Delta_{\nu}:=\left[\nu_{\min }, \nu_{\max }\right]$.

Finally we define the set

$$
\hat{\mathscr{H}}:=\left\{\hat{h} \in \mathcal{C}^{1}\left(\mathbb{R}_{+}, \hat{H}\right), \hat{h}^{\prime}: \mathbb{R}_{+} \rightarrow \hat{U}\right\}
$$

with $\hat{H}:=H+\Delta$ and $\hat{U}:=U+\Delta_{\nu}$.

According to the use of the main theorem, the statefeedback may take several forms: linear, polynomial or rational in $\hat{h}$ hence allowing a large variety of controllers.

The notations is as follows, for a symmetric matrices $A, B$, $A>B$ means $A-B$ is positive definite (i.e. $A-B>0$ ). For a square matrix $A$ we have $A^{H}=A+A^{T}$ where $A^{T}$ is the transpose of $A . \operatorname{Ker}(A)$ is a basis of the null-space of A. $A_{\perp}$ is the orthogonal complement of $A$ (i.e. $A^{T} A_{\perp}=0$ ). $\oplus$ is the direct sum of matrices: $A \oplus B=\left[\begin{array}{cc}A & 0 \\ 0 & B\end{array}\right] . \mathbb{K}^{n}$ is the set of skew-symmetric matrices of dimension $n$ and $\mathbb{S}_{++}^{n}$ is the cone of symmetric positive definite matrices of dimension $n . \mathcal{L}_{2}$ is the space of signals with finite energy (finite $\mathcal{L}_{2}$-norm): $\|f\|_{2}^{2}:=\int_{0}^{+\infty}|f(t)|^{2} d t<+\infty$ and $\mathcal{L}_{\infty}$ the space of signals with finite amplitude ( finite $\mathcal{L}_{\infty^{-}}$ norm): $\|f\|_{\infty}:=\max _{i}\left[\left|f_{i}(t)\right|\right]<+\infty$

The paper is organized as follows, section 2 develops preliminary results. Section 3 presents the new model transformation and the associated comparison system. Section 4 gives the delay-dependent stability test and comments on its use. Section 5 gives the main result of the paper, the delay-scheduled state-feedback existence theorem and its computation. Finally an illustrative example is presented in section 7 .

\section{PRELIMINARY RESULTS}

We provide here two results: the first one is used to relax a particular form of NMI while the second is a delaydependent stability lemma for system (1).

\subsection{Converting polynomial into linear dependence}

We provide here an useful result allowing to turn parameter dependent LMIs with polynomial dependence into a conservative linearly parametrized LMI.

We present first the following definition

Definition 2.1. A square matrix $S$ is said to be $\mathcal{S}^{2}$ structured if it writes $S=\left[S_{i j}\right]_{i, j}$ with blocks $S_{i j} \in$ $\mathbb{R}^{k \times k}, k>1$ and

$$
S_{i j}:= \begin{cases}0_{k \times k} & \text { if } i=j \\ S_{i j}=S_{j i}^{T} \in \mathbb{K}^{k} & \text { if } i \neq j\end{cases}
$$

It is now possible to express the linearization lemma:

Lemma 2.1. Suppose that a $\mathcal{M}(\delta)<0$ is a polynomially parametrized LMI in $\delta$ admitting a spectral factorization $\mathcal{M}(\delta)=U^{T}(\delta) \mathcal{N} U(\delta)<0$ where $U(\delta)$ is a basis of polynomials. Then $\mathcal{M}(\delta)<0$ if there exists a matrix $\mathcal{P}$ such that

$$
\mathcal{N}+\mathcal{R}+\mathcal{P} V(\delta)+V^{T}(\delta) \mathcal{P}^{T}<0
$$

where $V^{T}(\delta)=\operatorname{Ker}\left(U^{T}(\delta)\right)$ linear in $\delta$ and $\mathcal{R}$ is $\mathcal{S}^{2}$ structured.

Proof: The prof is a simple application of the creation lemma and projection lemma. First note that if we add the quadratic form $U^{T}(\delta) \mathcal{R} U(\delta)$ to $U^{T}(\delta) \mathcal{N} U(\delta)$ we do not change the expression of the quadratic form (due to skewsymmetric matrices) but this additional matrix modifies the eigenvalues and thus provides extra degrees of freedom. Apply the creation lemma on $U^{T}(\delta) \mathcal{N} U(\delta)<0$ leads to the existence of $\mathcal{Q}(\delta)$ such that

$$
\mathcal{N}+\mathcal{R}+\mathcal{Q}(\delta) V(\delta)+V^{T}(\delta) \mathcal{Q}(\delta)^{T}<0
$$

These parameter dependent LMIs are completely equivalent. Now note that $V(\delta)=V_{0}+\sum_{i=1}^{N_{p}} V_{i} \delta_{i}$ where $N_{p}$ is the number of distinct parameters. Then fixing $\mathcal{Q}$ to be parameter independent leads to the proposed result (7). $\square$ Remark 2.1. The matrix $\mathcal{R}$ is symmetric but is composed by blocks being skew-symmetric: e.g. $\mathcal{R}=\left[\begin{array}{cc}0 & \mathcal{R}_{1} \\ \mathcal{R}_{1}^{T} & 0\end{array}\right]$ with $\mathcal{R}_{1}+\mathcal{R}_{1}^{T}=0$. Adding such terms to a matrix, say $\mathcal{M}$, does modify the eigenvalue of the expression $\mathcal{M}+\mathcal{R}$ but does not change the value of the associated quadratic form. This is possible since the terms $U(\delta)$ are not column vectors but matrices with, generally, more than one column.

It is worth noting that we loose here the equivalence since we restrict $\mathcal{P}$ to be parameter independent. Nevertheless, this relaxation leads to quite good results. We can then use classical result for linearly parametrized LMIs such as multi-convexity or similar results. It is worth noting that the matrix $\mathcal{P}$ may have high dimensions due to the use of the spectral factorization. If ones uses convexity argument to conclude on negative definiteness of $\mathcal{M}(\delta)$ the number of LMIs to solve simultaneously will grow up exponentially w.r.t. the number of parameters $N_{p}$ and we 
get $\#(L M I s)=2^{N_{p}}$. In this case, it would be interesting to use the relaxation proposed in [Ben-Tal and Nemirovski, 2002] (and also presented in [Scherer])). This relaxation converts the set $2^{N_{p}}$ into one LMI with extra decision matrices. This is not detailed in the present paper and the readers should refer to cited articles to get more explanations.

A fundamental fact that we must point out concerns the relaxation of the parameter rational dependence. This type of LMIs can be turned into a polynomial dependence using the full-block $\mathcal{S}$-procedure and these ones can be then transformed into a linear dependence with lemma 2.1.

This result is highly related to the so-called SOS relaxations of parameter dependent LMIs. The main difference is that we take into account here the internal structure of the spectral factor. It follows that we can add constraints as used in SOS relaxations to give for instance relations between them and then turn the global inequality into a linear form.

\section{A NEW MODEL TRANSFORMATION}

We provide here a new model transformation allowing to turn a TDS with time-varying delays into an uncertain LPV system:

Let the operator

$$
\begin{aligned}
\mathcal{D}_{h}: \mathcal{L}_{2} & \rightarrow \mathcal{L}_{2} \\
\eta(t) & \rightarrow \frac{1}{\sqrt{h(t) h_{M}}} \int_{t-h(t)}^{t} \eta(s) d s
\end{aligned}
$$

This operator enjoys the following properties:

(1) $\mathcal{D}_{h}$ is $\mathcal{L}_{2}-\mathcal{L}_{2}$ stable.

(2) $\mathcal{D}_{h}$ has an induced $\mathcal{L}_{2}-\mathcal{L}_{2}$ norm lower than 1.

Proof: Let us prove first that for a $\mathcal{L}_{2}$ input signal we obtain a $\mathcal{L}_{2}$ output signal effectively. Assume that $\eta(t)$ is continuous and denote by $\eta_{p}(t)$ the signal satisfying $d \eta_{p}(t) / d t=\eta(t)$ then we have

$$
\mathcal{D}_{h}(\eta(t))=\frac{\eta_{p}(t)-\eta_{p}(t-h(t))}{\sqrt{h(t) h_{M}}}
$$

Note that if $h(t)$ is always positive then (9) is bounded since $\eta(t)$ belongs to $\mathcal{L}_{2}$ (and hence to $\mathcal{L}_{\infty}$ ). The main problem is when the delay attains 0. Suppose now that there exist a (possibly infinite) family of time instants $t_{i+1}>t_{i} \geq 0$ such that we have $h\left(t_{i}\right)=0$. It is obvious that $\eta_{p}(t)$ is continuously differentiable and hence we have

$$
\lim _{t \rightarrow t_{i}} \sqrt{\frac{h(t)}{h_{M}}} \frac{\eta_{p}(t)-\eta_{p}(t-h(t))}{h(t)}=0
$$

As $\eta(t)$ is continuous and belongs to $\mathcal{L}_{2}$, we can state that $\eta\left(t_{i}\right)$ is always finite and then the output signal remains bounded even if the delay reaches zero. We have proved that $\mathcal{D}_{h}$ has a finite $\mathcal{L}_{\infty}$-induced norm (no singularities). To prove that it has a finite induced $\mathcal{L}_{2}$-norm it suffices to compute its $\mathcal{L}_{2}-\mathcal{L}_{2}$ gain defined by $\left\|\mathcal{D}_{h}(\eta)\right\|_{2} /\|\eta\|_{2}$.

$\left\|\mathcal{D}_{h}(\eta)\right\|_{2}^{2}:=\int_{0}^{+\infty} \frac{d t}{h_{M} h(t)} \int_{t-h(t)}^{t} \eta^{T}(\theta) d \theta \cdot \int_{t-h(t)}^{t} \eta(\theta) d \theta$

Then applying the Jensen's inequality (see $[\mathrm{Gu}$ et al., 2003]) and bounding the integral with $h_{M}$ we obtain

$$
\left\|\mathcal{D}_{h}(\eta)\right\|_{2}^{2} \leq \int_{0}^{+\infty} \frac{d t}{h_{M}} \int_{t-h_{M}}^{t} \eta^{T}(\theta) \eta(\theta) d \theta
$$

Then exchanging the order of integration and considering zero initial condition leads to the inequality

$$
\left\|\mathcal{D}_{h}(\eta)\right\|_{2}^{2} \leq \int_{0}^{+\infty} \eta^{T}(t) \eta(t) d t=\sqrt{h_{M}}\|\eta\|_{2}^{2}
$$

We have then proved that $\mathcal{D}_{h}$ defines a $\mathcal{L}_{2}-\mathcal{L}_{2}$ stable operator with an $\mathcal{L}_{2}$-induced norm lower than 1 .

We show now how to use this operator to transform a timedelay system into a uncertain LPV system. Consider system $(1)$ and note that $x_{h}(t)=x(t)-\left(h(t) / h_{M}\right)^{1 / 2} \mathcal{D}_{h}(\dot{x}(t))$ then substituting into system (1) and once expressed into a LFT form we obtain then

$$
\begin{aligned}
& \dot{y}(t)=\bar{A} y(t)-\left(h(t) h_{M}\right)^{1 / 2} A_{h} w_{0}(t)+B_{u} u(t)+E w(t) \\
& z_{0}(t)=\dot{y}(t) \\
& z(t)=\bar{C} y(t)-\left(h(t) h_{M}\right)^{1 / 2} C_{h} w_{0}(t)+D_{u} u(t)+E w(t) \\
& w_{0}(t)=\mathcal{D}_{h}\left(z_{0}(t)\right)
\end{aligned}
$$

with $\bar{A}=A+A_{h}$ and $\bar{C}=C+C_{h}$. We have changed the state denomination since systems (1) and (10) may be not equivalent (on the other hand we keep the same name for input/output signals for sake of simplicity). Indeed, the model transformation creates additional dynamics inducing conservatism (see [Gu et al., 2003]) and then an inequivalence between both models.

This system is then obviously:

- uncertain due to the presence of the "unknown" structured norm bounded LTV dynamic operator $\mathcal{D}_{h}$ and for this part we will use results of robust stability analysis and robust synthesis.

- parameter varying (even affine in $\sqrt{h(t)}$ ) and we will use parameter dependent Lyapunov functions to tackle this time-varying part.

It is clear that this system is not equivalent to (1) due to the model transformation adding additional dynamics (see [Gu et al., 2003]) but is not detailed here for sake of brevity. Just note that additional dynamics may be a source of conservatism in stability analysis. Nevertheless, in the stabilization problem this is less problematic since we aim to stabilize the system and hence we stabilize these additional dynamics (assuming they are stabilizable).

\section{DELAY-DEPENDENT STABILITY}

We present here our result of delay dependent stability based on the model transformation presented in section 3 . Lemma 4.1. System (10) without control input (i.e. $u(t)=$ $0)$ is asymptotically stable for $h \in \mathscr{H}$ and satisfies the $\mathcal{H}_{\infty}$-norm property $\|z\|_{2} /\|w\|_{2}<\gamma(h, \dot{h})$ if there exist a smooth matrix function $P: H \rightarrow \mathbb{S}_{++}^{n}$, matrix functions $D: H \times U \rightarrow \mathbb{S}_{++}^{n}, G: H \times U \rightarrow \mathbb{K}^{n}$ and a function $\gamma: H \times U \rightarrow \mathbb{R}_{++}$such that the LMI (11) holds for all $h \in H$ and $\dot{h} \in U$ :

Proof: Consider the parameter dependent Lyapunov function with parameter dependent supply rate $s(\cdot, \cdot, \cdot, \cdot)$ 


$$
\left[\begin{array}{ccccc}
{\left[\bar{A}^{T} P(h)\right]^{H}+\frac{d P}{d h} \dot{h}} & -\left(h h_{M}\right)^{1 / 2} P(h) A_{h}+\bar{A}^{T} G(h, \dot{h}) & P(h) E & \bar{C}^{T} & \bar{A}^{T} D(h, \dot{h}) \\
\star & -D(h)-\left[\left(h h_{M}\right)^{1 / 2} A_{h}^{T} G(h, \dot{h})\right]^{H} & G^{T}(h, \dot{h}) E & -h C_{h}^{T} & -\left(h h_{M}\right)^{1 / 2} A_{h}^{T} D(h, \dot{h}) \\
\star & \star & -\gamma(h, \dot{h}) I_{p} & F^{T} & E^{T} D(h, \dot{h}) \\
\star & \star & \star & -\gamma(h, \dot{h}) I_{q} & 0 \\
\star & \star & \star & \star & -D(h, \dot{h})
\end{array}\right]<0
$$

$$
V:=x^{T}(t) P(h(t)) x(t)-\int_{0}^{t} s(w(s), z(s), h(s), \dot{h}(s)) d s
$$

where $P\left(h(t)=\sum_{i=1}^{N} P_{i} h(t)^{i}\right.$ and

$$
\begin{aligned}
s(\cdot, \cdot, \cdot, \cdot):= & \gamma(h(t), \dot{h}(t)) w^{T}(t) w(t) \\
& +\gamma^{-1}(h(t), \dot{h}(t)) z^{T}(t) z(t)
\end{aligned}
$$

Then consider system

$$
\begin{aligned}
& \dot{x}(t)=\bar{A} x(t)-\left(h(t) h_{M}\right)^{1 / 2} A_{h} \mathcal{D}_{h}(\dot{x}(t))+E w(t) \\
& z(t)=\bar{C} x(t)-\left(h(t) h_{M}\right)^{1 / 2} C_{h} \mathcal{D}_{h}(\dot{x}(t))+F w(t)
\end{aligned}
$$

with $\bar{A}=A+A_{h}$ and $\bar{C}=C+C_{h}$. Computing the derivative of the parameter dependent Lyapunov function (12) along trajectories solutions of the system (14) leads to

$$
\begin{aligned}
\dot{V}= & x^{T}(t) \dot{h}(t) \frac{\partial P}{\partial h} x(t)+\left[\dot{x}^{T}(t) P(h(t)) x(t)\right]^{H}+\gamma w^{T}(t) w(t) \\
& +\gamma^{-1}\left(\bar{C} x(t)-h(t) C_{h} \mathcal{D}_{h}(\dot{x}(t))+F w(t)\right) z^{T}(t)
\end{aligned}
$$

Then, using the so-called full-block $\mathcal{S}$-procedure, this rewrites as

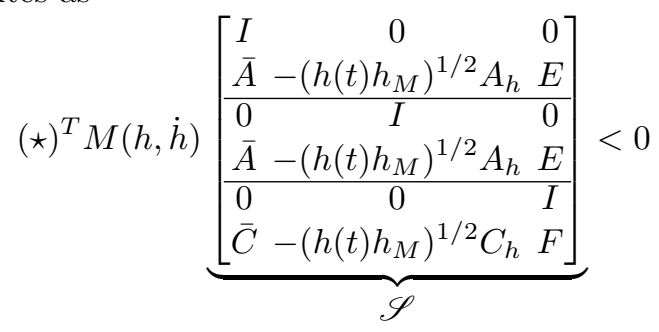

where $M(h, \dot{h})=\left[\begin{array}{cc}\dot{h} \frac{d P(h)}{d h} & P(h) \\ P(h) & 0\end{array}\right] \oplus \mho(h, \dot{h}) \oplus\left[-\gamma(h, \dot{h}) I_{p}\right] \oplus$ $\left[\gamma^{-1}(h, \dot{h}) I_{q}\right]$ and $\left.\mho(h, \dot{h})\right)$ satisfies

$$
\left[\begin{array}{c}
\mathcal{D}_{h} \\
I_{n}
\end{array}\right]^{T} \mho(h, \dot{h})\left[\begin{array}{c}
\mathcal{D}_{h} \\
I_{n}
\end{array}\right]>0
$$

The separator $\mho(h, \dot{h})=\mho^{*}(h, \dot{h})$ is chosen following the following facts:

- As $\left\|\mathcal{D}_{h}\right\|_{\infty}<1$ then $\mathcal{D}_{h}$ may satisfy

$$
\left[\begin{array}{c}
\mathcal{D}_{h} \\
I_{n}
\end{array}\right]^{T} \underbrace{\left[\begin{array}{cc}
-1 & 0 \\
0 & 1
\end{array}\right]}_{\mho_{1}}\left[\begin{array}{c}
\mathcal{D}_{h} \\
I_{n}
\end{array}\right]>0
$$

- As the uncertain term is scalar and is repeated diagonally of a number equalling the state dimension then we have

$$
\left[\begin{array}{c}
\mathcal{D}_{h} \\
I_{n}
\end{array}\right]^{T} \underbrace{\left[\begin{array}{cc}
0 & -i \\
i & 0
\end{array}\right]}_{\mathcal{\mho}_{2}}\left[\begin{array}{c}
\mathcal{D}_{h} \\
I_{n}
\end{array}\right]=0
$$

Hence a set of separators are parametrized as $v=v_{1} \otimes$ $D+\mho_{2} \otimes G$ where $D=D^{*}>0$ and arbitrary $G=G^{*}$. But as we restrict the set of separators to be hermitian and the signal are are real valued then the separator becomes

$$
\mho(h, \dot{h}):=\left[\begin{array}{cc}
-D(h, \dot{h}) & G^{T}(h, \dot{h}) \\
\star & D(h, \dot{h})
\end{array}\right]
$$

where $D: H \times U \rightarrow \mathbb{S}_{++}^{n}$ and $G: H \times U \rightarrow \mathbb{K}^{n}$.

Then expand (16) and perform a Schur complement on quadratic term

$-\left[\begin{array}{cc}\bar{C}^{T} & \bar{A}^{T} Q(h, \dot{h}) \\ -h_{r}^{1 / 2} C_{h} & -h_{r}^{1 / 2} A_{h} Q(h, \dot{h}) \\ F & E^{T} Q(h, \dot{h})\end{array}\right]\left[\begin{array}{cc}-\gamma^{-1}(h, \dot{h}) I_{q} & 0 \\ 0 & -Q^{-1}(h, \dot{h})\end{array}\right](\star)^{T}$

where $h_{r}=h h_{M}$ leads to inequality (11).

\section{DELAY-SCHEDULED STATE-FEEDBACK DESIGN}

We provide in that section the computation of a delayscheduled state-feedback of the form (4). In this case, the closed-loop system is then given by

$$
\begin{aligned}
& \dot{y}(t)=\bar{A}_{c l}(\hat{h}, \dot{\hat{h}}) y(t)-A_{h}\left(h(t) h_{M}\right)^{1 / 2} w_{0}(t)+E w(t) \\
& z(t)=\bar{C}_{c l}(\hat{h}, \hat{\hat{h}}) y(t)-C_{h}\left(h(t) h_{M}\right)^{1 / 2} w(t)+F w(t) \\
& z_{0}(t)=\dot{y}(t) \\
& w_{0}(t)=\mathcal{D}_{h}\left(z_{0}(t)\right)
\end{aligned}
$$

with a state feedback of the form $K\left(h+\delta_{h}\right), \bar{A}_{c l}\left(h, \delta_{h}\right)=$ $A+B_{u} K(\hat{h}, \dot{\hat{h}})$ and $\bar{C}_{c l}\left(h, \delta_{h}\right)=C+D_{u} K(\hat{h}, \dot{\hat{h}})$

There exist several ways to compute this controller:

(1) Use a change of variable and in this case it is possible to fix a desired form to the controller.

(2) Elaborate stabilizability test and deduce from it a suitable controller either by explicit formulae or by optimization.

In the present paper we propose a solution based on a change of variable. It has the great benefits of being more simple than the second approach and of fixing the complexity degree of the controller. Nevertheless, the second approach is really interesting since it provides explicit solutions for the controller but these solutions may not be implementable since some value (as the delay derivative) are not accessible. Fixing some matrices to be independent of parameters solves this problem but raises the conservatism of the approach.

Theorem 5.1. The system (10) is stabilizable with a delayscheduled state feedback $K(\hat{h})=Y(\hat{h}) X^{-1}(\hat{h})$ if there exists a smooth matrix function $X: \hat{H} \rightarrow \mathbb{S}_{++}^{n}$, matrix functions $\tilde{D}: H \times U \times \hat{H} \times \hat{U} \rightarrow \mathbb{S}_{++}^{n}, \tilde{G}: H \times U \times \hat{H} \times$ $\hat{U} \rightarrow \mathbb{K}^{n}$ and a scalar function $\gamma: H \times U \times \hat{H} \times \hat{U} \rightarrow \mathbb{R}_{++}$ such that the LMI (22) holds for all $h \in H, \dot{h} \in U, \delta \in \Delta$ and $\dot{\delta} \in \Delta_{\nu}$, where $\xi=\operatorname{col}\left(h, \delta_{h}, \dot{h}, \dot{\delta_{h}}\right)$ and $h_{r}=h h_{M}$.

Proof: 


$$
\left[\begin{array}{ccccc}
-\dot{\hat{h}} \frac{\partial X(\hat{h})}{\partial \hat{h}}+\left[X(\hat{h}) \bar{A}^{T}+Y^{T}(\hat{h}) B_{u}^{T}\right]^{H} & X(\hat{h}) \bar{C}^{T}+Y^{T}(\hat{h}) D_{u}^{T} & -\dot{\hat{h}} \frac{\partial X(\hat{h})}{\partial \hat{h}}+\bar{A} X(\hat{h})+h_{r}^{1 / 2} A_{h} \tilde{G}^{T}(\xi) & h_{r}^{1 / 2} A_{h} \tilde{D}(\xi) & E \\
\star & -\gamma(\xi) I_{q} & \left(h / h_{M}\right)^{1 / 2} C_{h} \tilde{G}^{T}(\xi)+\bar{C} X(h) & h_{r}^{1 / 2} C_{h} \tilde{D}(\xi) & F \\
\star & \star & -\dot{\hat{h}} \frac{\partial X(\hat{h})}{\partial \hat{h}}-\tilde{D}(\xi) & 0 & 0 \\
\star & \star & \star & -\tilde{D}(\xi) & 0 \\
\star & \star & \star & \star & -\gamma(\xi) I_{p}
\end{array}\right]
$$

First note that the real delay is $h(t)$ and the known one is $\hat{h}(t)=h(t)+\delta_{h}(t)$. Moreover as we use the change of variable approach then $X$ must depend on $\hat{h}(t)$ only. Nevertheless, the other variable may depend on all the parameters (i.e. $\left.h(t), \delta_{h}(t), \dot{h}(t), \dot{\delta}_{h}(t)\right)$. From here let $\xi=$ $\operatorname{col}\left(h, \delta_{h}, \dot{h}, \dot{\delta_{h}}\right)$ for simplicity.

Consider the inequality (16) as a departure point, where we inject the closed-loop system expression. Note that $n^{-}(M)=2 n+m$ (where $n^{-}(M)$ denotes the number of strictly negative eigenvalues of $M$ ) and equals the rank of the subspace $\mathscr{S}$ (defined in (16)) then it is possible to apply the dualization lemma (see [Scherer et al., 1997, Scherer and Weiland, 2004]) and we obtain

$$
(\star)^{T} M^{-1}(\xi)\left[\begin{array}{ccc}
-\bar{A}_{c l}^{T}(\hat{h}) & -\bar{C}^{T}(\hat{h}) & 0 \\
I_{n} & 0 & I_{n} \\
\hline\left(h h_{M}\right)^{1 / 2} A_{h}^{T} & \left(h h_{M}\right)^{1 / 2} C_{h}^{T} & 0 \\
0 & 0 & -I_{n} \\
\hline-E^{T} & -F^{T} & 0 \\
0 & I_{q} & 0
\end{array}\right]>0
$$

where $M^{-1}(\xi)=\left[\begin{array}{cc}\frac{d P(\hat{h})}{d t} & P(\hat{h}) \\ \star & 0\end{array}\right]^{-1} \oplus \mho^{-1}(\xi) \oplus\left[-\gamma^{-1}(\xi)\right] \oplus$ $[\gamma(\xi)]$. Let $X=P^{-1}$ and then $\frac{d X(\hat{h})}{d t}=-X \frac{d P(\hat{h})}{d t} X$, we have $\left[\begin{array}{cc}\frac{d P(\hat{h})}{d t} & P(\hat{h}) \\ \star & 0\end{array}\right]^{-1}=\left[\begin{array}{cc}0 & X(\hat{h}) \\ \star & \frac{d X(\hat{h})}{d t}\end{array}\right]$. Denote also $\mho^{-1}(\xi)=\left[\begin{array}{cc}-\tilde{D}(\xi) & \tilde{G}^{T}(\xi) \\ \star & \tilde{D}(\xi)\end{array}\right]$ with $\tilde{D} \in \mathbb{S}_{++}^{n}$ and $\tilde{G} \in \mathbb{K}^{n}$ Moreover $\mho^{-1}(\cdot)$ satisfies the inequality

$$
\left[\begin{array}{c}
-I_{n} \\
\mathcal{D}_{h}^{T}(\cdot)
\end{array}\right]^{T}\left[\begin{array}{cc}
-\tilde{D}(\xi) & \tilde{G}^{T}(\xi) \\
\star & \tilde{D}(\xi)
\end{array}\right]\left[\begin{array}{c}
-I_{n} \\
\mathcal{D}_{h}^{T}(\cdot)
\end{array}\right]<0
$$

Then expand (23), multiply it by -1 and considering $\tilde{R}(h, \dot{h})<0$, this allows to use a Schur complement on the quadratic term:

$$
-\left[\begin{array}{ccc}
\left(h h_{M}\right)^{1 / 2} A_{h} \tilde{D} & E \\
\left(h h_{M}\right)^{1 / 2} C_{h} \tilde{D} & F \\
0 & 0
\end{array}\right]\left[\begin{array}{cc}
-\tilde{D}^{-1} & 0 \\
0 & -\gamma^{-1}(\xi) I_{p}
\end{array}\right](\star)^{T}
$$

and we obtain the inequality $(22)$ where $Y(\hat{h})=K(\hat{h}) X(\hat{h})$.

\section{RELAXATION OF PARAMETRIZED LMIS AND PARAMETRIZED OBJECTIVES}

We have express the stability and stabilizability problems as polynomially parametrized LMIs. Moreover the $\mathcal{H}_{\infty}-$ norm is expressed as a positive function of the parameters and its minimization is not a well-defined problem. We detail in that section how to equivalently relax these inequalities and objective cost.

\subsection{Equivalent Relaxations of parameter dependent LMIs}

Note that any parameter dependent LMIs can be rewritten in a spectral form

$$
U^{T}(\xi) Z U(\xi)<0
$$

where $\xi$ represent the vector of parameters. Hence it is possible to use lemma 2.1 to linearize the dependence onto the parameters and we get for any matrix $W$ of appropriate dimensions and a $\mathcal{S}^{2}$-structured matrix $\tilde{Z}$

$$
Z+\tilde{Z}+W V(\xi)+V^{T}(\xi) W^{T}<0
$$

where $V(\xi) U(\xi)=0$ and $V(\xi)$ is linear in $\xi$. Then this can be relaxed using a convex argument: it suffices to verify the latter inequality at each vertices of the polytope where $\xi$ evolves to prove the negativity of the LMI for all parameter values. That type of relaxation must be used on the LMIs, parameterized decisions matrices $(X, \gamma, D)$.

We have now turned parameter dependent LMIs into a set of parameter independent LMIs.

\subsection{Costs}

As $\gamma(\xi)$ is polynomial then there exists several costs $\mathcal{J}(\cdot)$ according to the application, but is is possible to give a general formulation:

$$
\mathcal{J}_{\theta}(\gamma):=\int_{H \times U \times \bar{H} \times \hat{U}} \theta(\xi) \gamma(\xi) d \xi
$$

with $\int_{H \times U \times \bar{H} \times \hat{U}} \theta(\xi) d \xi=1$.

We propose here some interesting values of the weighting function $\theta(\cdot, \cdot)$ :

- $\theta_{1}(\xi)=\mu(H \times U \times \bar{H} \times \hat{U})^{-1}$ where $\mu(\cdot)$ is the Lebesgue measure.

- $\theta_{2}(\xi)=\delta\left(\prod_{i=1}^{f}\left(\xi-\xi_{i}\right)\right)$ with $\delta(t)$ is the Dirac distribution.

- $\theta_{3}(\xi)=p(\xi)$ where $p(\cdot)$ denotes for instance a probability density function.

The first one minimizes the volume below the surface $\gamma: H \times U \times \bar{H} \times \hat{U} \rightarrow \mathbb{R}_{+}$without any preferences. The second one aims to minimize the $\mathcal{H}_{\infty}$-norm for certain delay, errors and their derivative values for systems (may be interesting for discrete valued delay. The third one is dedicated when we have a stochastic model of the delay (and eventually a model for its derivative) attempts for instance to minimize in priority the $\mathcal{H}_{\infty}$-norm for high probable delay values. 


\begin{tabular}{l|c} 
controller & $\gamma$ \\
\hline constant & 19.4055 \\
linear & 19.4055 \\
rational & 15.5030
\end{tabular}

Table 1. Minimal $\mathcal{H}_{\infty}$-norm of the closed-loop system

\section{EXAMPLE}

We aim to stabilize the following time delay system with time-varying delay

$$
\begin{aligned}
\dot{x}(t)= & {\left[\begin{array}{ll}
11 & 23 \\
14 & 16
\end{array}\right] x(t)+\left[\begin{array}{ll}
15 & 18 \\
12 & 23
\end{array}\right] x_{h}(t)+\left[\begin{array}{c}
12 \\
0
\end{array}\right] u(t) } \\
& +\left[\begin{array}{l}
11 \\
22
\end{array}\right] w(t) \\
z(t)= & {\left[\begin{array}{ll}
1 & 0 \\
0 & 0
\end{array}\right] x(t)+\left[\begin{array}{c}
0 \\
0.1
\end{array}\right] u(t) }
\end{aligned}
$$

with $h(t) \in[0,5],|\dot{h}| \leq 0.2, \dot{\delta}, \delta \in[-0.1,0.1]$. We choose a constant $\gamma>0$ and constant scaling (i.e. constant $\tilde{D}, \tilde{G})$. We compute three different controllers: a constant feedback, a linear state-feedback (i.e. $K(\hat{h})=K_{0}+$ $\left.K_{1} \hat{h}\right)$ and a rational state-feedback (i.e. $K(\hat{h})=\left(K_{n 0}+\right.$ $\left.\left.K_{n 1} \hat{h}\right)\left(K_{d 0}+K_{d 1} \hat{h}\right)^{-1}\right)$. The results are summarized in table 1 . We can see that in this example, taking a constant or linearly dependent controller does not lead to a better closed-loop $\mathcal{H}_{\infty}$-norm. Nevertheless, taking a rationally dependent controller leads to a better $\mathcal{H}_{\infty}$-norm for the closed-loop system. This happens for two main reasons:

(1) The controller have a more complex form

(2) Through the use of a parameter dependent Lyapunov matrix, the information on the delay-derivative is embedded and then reduce the conservatism.

\section{CONCLUSION}

We have presented in that paper a new model transformation refining the one in [Briat et al., 2007]. It allows to consider a wider class of delay values and similarly turns a time-delay system into an uncertain LPV system where the delay acts now as a time-varying parameter. Based on that description it is possible to propose a new delay-dependent stability lemma based on the full-block $\mathcal{S}$-procedure and derive a constructive approach to the solution of a stabilizing state-feedback. Both constant and delay-scheduled controllers are considered and uncertainties on the knowledge of the delay are taken a priori into account in the synthesis problem. All the results are given in terms of parameter dependent LMIs with polynomial dependence onto parameters. These parametrized LMIs are exactly relaxed into a set of LMIs using a result exposed in this paper which seems to be new. Finally we show the effectiveness of the approach trough an academic example.

\section{REFERENCES}

P. Apkarian and R.J. Adams. Advanced gain-scheduling techniques for uncertain systems. IEEE Transactions on Automatic Control, 6:21-32, 1998.

A. Ben-Tal and A. Nemirovski. On tractable approximmations of uncertain linear matrix inequalities affected by interval uncertainty. SIAM J. Optim, 12(3):811-833, 2002.

C. Briat, O. Sename, and J-F. Lafay. A LFT $/ \mathcal{H}_{\infty}$ statefeedback design for linear parameter varying time delay systems. In European Control Conference 2007, Kos, Greece, 2007.

S. G. Dietz, C. W. Scherer, and W. Huygen. Linear parameter-varying controller synthesis using matrix sum-of squares. In Proceedings of the XII LatinAmerican congress on automatic control, 2006.

E. Fridman. Stability of systems with uncertain delays: a new 'complete' lyapunov-krasovskii functional. IEEE Transactions on Automatic Control, 51:885-890, 2006.

F. Gouaisbaut and D. Peaucelle. Stability of time-delay systems with non-small delay. In Conference on Decision and Control, San Diego, California, 2006a.

F. Gouaisbaut and D. Peaucelle. Delay depependent robust stability of time delay-systems. In $5^{\text {th }}$ IFAC Symposium on Robust Control Design, Toulouse, France, 2006b.

K. Gu, V.L. Kharitonov, and J. Chen. Stability of TimeDelay Systems. Birkhäuser, 2003.

C. Y. Kao and A. Rantzer. Stability analysis of systems with uncertain time-varying delays. Automatica, 43: 959-970, 2007.

S.-I. Niculescu. Delay effects on stability. A robust control approach, volume 269. Springer-Verlag: Heidelbeg, 2001.

M. Roozbehani and C. R. Knospe. Robust stability and $\mathcal{H}_{\infty}$ performance analysis of interval-dependent time-delay system. In American Control Conference, Portland, USA, 2005.

C. Scherer. Robust mixed control and LPV control with full block scalings. Advances in LMI Methods in Control, SIAM, 1999.

C. Scherer and S. Weiland. Linear matrix inequalities in control. Technical report, Delft Center for Systems and Control (Delft University of Technology) and Department of Electrical Engineering (Eindhoven University of Technology), 2004.

C. Scherer, P. Gahinet, and M. Chilali. Multiobjective output-feedback control via lmi optimization. IEEE Transaction on Automatic Control, 42(7):896-911, 1997. C. W. Scherer. LMI relaxations in robust control. Preprint.

C. W. Scherer and C. W. J. Hol. Matrix sum-of-squares relaxations for robust semi-definite programs. Mathematical Programming Series B, 107:189-211, 2006.

O. Sename, J.F. Lafay, and R. Rabah. Controllability indices of linear systems with delays. KybernetiKa, 6: 559-580, 1995.

V. Suplin, E. Fridman, and U. Shaked. $\mathcal{H}_{\infty}$ control of linear uncertain time-delay systems - a projection approach. IEEE Transactions on Automatic Control, 51:680-685, 2006.

E. Witrant, D. Georges, C. Canudas De Wit, and O. Sename. Stabilization of network controlled systems with a predictive approach. In $1^{\text {st }}$ Workshop on Networked Control System and Fault Tolerant Control, Ajaccio, France, 2005.

J. Zhang, C.R. Knospe, and P. Tsiotras. Stability of timedelay systems: Equivalence between lyapunov and scaled small-gain conditions. IEEE Transactions on Automatic Control, 46:482-486, 2001. 